\title{
Activation tagging of the LEAFY PETIOLE gene affects leaf petiole development in Arabidopsis thaliana
}

\author{
Eric van der Graaff ${ }^{1, *}$, Amke Den Dulk-Ras ${ }^{2}$, Paul J. J. Hooykaas ${ }^{2}$ and Beat Keller ${ }^{1}$ \\ ${ }^{1}$ Institute of Plant Biology, University of Zürich, Zollikerstrasse 107, CH-8008 Zürich, Switzerland \\ ${ }^{2}$ Institute of Molecular Plant Sciences, Leiden University, Clusius Laboratory, Wassenaarseweg 64, 2333 AL Leiden, The \\ Netherlands \\ *Author for correspondence (e-mail: egraaff@botinst.unizh.ch)
}

Accepted 31 August; published on WWW 24 October 2000

\section{SUMMARY}

In a screen for leaf developmental mutants we have isolated an activator T-DNA-tagged mutant that produces leaves without a petiole. In addition to that leafy petiole phenotype this lettuce (let) mutant shows aberrant inflorescence branching and silique shape. The $L E A F Y$ $P E T I O L E$ ( $L E P$ ) gene is located close to the right border of the T-DNA insert linked with these dominant phenotypes and encodes a protein with a domain with similarity to the DNA binding domain of members of the AP2/EREBP family of transcription factors. Introduction of the activation-tagged $L E P$ gene in wild-type plants conferred all the phenotypic aberrations mentioned above. The leafy petiole phenotype consists of a conversion of the proximal part of the leaf from petiole into leaf blade, which means that leaf development in let is disturbed along the proximodistal axis. Therefore, LEP is involved in either cell division activity in the marginal meristem or patterning along the proximodistal axis.

Key words: LEAFY PETIOLE, lettuce, Marginal meristem, Petiole development, Leaf blade, Arapidopsis thaliana

\section{INTRODUCTION}

The development of dicotyledonous leaves is a complicated process involving regulated division and expansion of cells (Tsukaya et al., 1994; Tsukaya, 1995), resulting in the patterning of a dorsoventral and proximodistal axis of asymmetry in the leaf primordium (Van Lijsebettens and Clarke, 1998; Poethig, 1997). The characterisation of the phantastica (phan) mutant of snapdragon (Waites and Hudson, 1995; Waites et al., 1998) and the phabulosa mutant of Arabidopsis thaliana (McConnell and Barton, 1998) showed that the establishment of dorsoventrality in the leaf primordium is an essential step for the initiation of marginal meristem activity leading to leaf blade formation. Although PHAN is necessary for the establishment of dorsoventrality, PHAN expression was observed throughout the whole leaf primordium. Therefore, it was proposed that PHAN interacts with other genes that show spatially restricted expression patterns or that PHAN activity is inhibited by a factor exhibiting specific expression in the ventral domain (Waites et al., 1998). Other genes that have been shown to be involved in the specification of dorsoventrality are members of the $Y A B B Y$ family of transcription factors specifying ventral cell fate and PINHEAD/ZWILLE (PNH) specifying dorsal cell fate (Bowman, 2000 and references therein). Interestingly, both the $Y A B B Y$ genes and $P N H$ first show expression throughout the very young leaf primordium before their expression becomes restricted to the ventral or dorsal domain, respectively.
Therefore, it remains to be determined which genes are primarily responsible for the establishment of dorsoventrality.

In contrast to the number of mutants isolated affecting dorsoventral patterning only a few mutants have been isolated that are disturbed in the other patterning processes taking place in the leaf primordium. In the lam-1 mutant of Nicotiana sylvestris (McHale, 1992, 1993) the blade initiation site is established normally, but subsequent cell division is disturbed (McHale and Marcotrigiano, 1998). LAM was shown to act after the establishment of dorsoventrality in the leaf primordium induced by PHAN and probably encodes a noncell-autonomous dorsalising factor necessary for maintaining dorsoventrality in the developing leaf blade (McHale and Marcotrigiano, 1998). The Arabidopsis arrested development 3 (add3) mutant (Pickett et al., 1996) displays defects in the proper formation of a leaf blade and $A D D 3$ could be a gene with similar function to $L A M$.

To come to a better understanding of leaf development, we generated a pool of Arabidopsis lines harbouring an activator T-DNA construct (van der Graaff and Hooykaas, 1996; van der Graaff et al., 1996) that was screened for leaf developmental mutants. Here, we describe the phenotypic and genetic analysis of the dominant T-DNA-tagged lettuce (let) mutant, which exhibited a novel type of defect in leaf development. The mutant leaves lack a petiole and consequently, the leaves consist of a large leaf blade. This leafy petiole phenotype is the result of the conversion of petiole into leaf blade. We show that this altered leaf development is caused by activation tagging of 
$L E P$, a gene that codes for a protein with similarity to the family of AP2/EREBP like transcription factors.

\section{MATERIALS AND METHODS}

\section{Growth conditions and plant transformations}

Seeds of the transgenic lines and wild-type control (all Arabidopsis thaliana ecotype C24) were either surface sterilised and grown in tissue culture on 1/2MS10 medium (van der Graaff and Hooykaas, 1996) or in soil under a $16 / 8$ hours light/dark regime at $21^{\circ} \mathrm{C}$. The pool of 550 transgenic lines harbouring the activator construct pSDM1550 was generated using a leaf transformation protocol as described before (van der Graaff and Hooykaas, 1998). The 35SDE$L E P$-mac12.6 and 35SDE-LEP constructs (Fig. 1B) were transformed to Arabidopsis using the root transformation protocol described by Vergunst et al. (1998) and the DE-LEP (Fig. 1B) and cDNAoe constructs were transformed using the floral dip method (http://www.cropsci.uiuc.edu/ a-bent/protocol.html). The cDNAoe construct was obtained by cloning the PCR-amplified LEP coding region ( $\mathrm{pLEPcodF}$ and $\mathrm{pLEPcodR}$ primers) in the $35 \mathrm{~S}$ overexpression cassette of pART7 (Gleave, 1992).

\section{Genetic analysis of the let mutant}

The original let mutant was isolated as a tetraploid transgenic line harbouring two independently segregating T-DNA loci. This mutant line was backcrossed two consecutive times with wild-type plants to obtain diploid, single locus lines. The (sub)lines resulting from these crosses all segregated for a single T-DNA locus and were diploid. The lines segregating for the dominant leafy petiole phenotype were used for linkage analysis. All seedlings exhibiting the leafy petiole phenotype in the progeny of these lines were hygromycin resistant (>200 seedlings) and all these lines harboured the same T-DNA insert in Southern blot analysis. Therefore, the leafy petiole phenotype was closely linked with this T-DNA insert. The isolation of the T-DNA insert with flanking plant DNA (Fig. 1A) and subsequent sequence analysis was performed as described by van der Graaff and Hooykaas (1996).

\section{Primers}

The following primers were used in this study (Fig. 1A). RTL1F: (41283) 5'-TACTCAGACATGCCTCCT-3', RTL1R: (41529) 5'-AGCTCATTACTACTGTCA-3', RTL2F: (42337) 5'-GATAAATGGGCAGAGCGT-3', RTL2R: (42733) 5'-CAATAGGACTGAGAAAAGGT-3', GapCF 5'-AGCTCGTCGCTGTCAACG-3', GapCR: 5'-GACAGCCTTGGCAGCTCCT-3', 35SleaderF: 5'-TTCTTTCAAATACTTCCAC-3', pLETF: (40363) 5'-GTATCTAAGGTAACATGCT-3', pLETR: (40804) 5'-GTGGCCCATGATATATCA-3', LETcodF: (41052) 5'-TTACCATGGGAAAGATGAACACAACA-3', LETcodR: (41690) 5'-AGACTGCAGATTAGGAGCCAAAGTA-3', LEP5'race: (41607) 5'-GACGAGTAGTCGTCACCGGTCCAG-3', LEP3'race: (41294) 5'-TGCCTCCTTCCTCATCCGTCACC-3' and PROM5'race: (40980) 5'-GAAGGAAGAGAGGGAATGTGTGTTGG-3'. The numbers in brackets indicate the position of the primers on the BAC clone MAC12.

\section{RNA isolation and analysis of gene expression}

Total RNA was isolated from 2-week old plants grown in liquid culture (1/2MS10 medium) according to van Slogteren et al. (1983), unless otherwise stated. Northern analysis was performed as described in Memelink et al. (1994). Poly(A) ${ }^{+}$RNA was isolated using the Oligotex mRNA kit (Qiagen). Transcripts were detected using [ $\alpha$ ${ }^{32}$ P]dCTP random labelled probes. To test for equal loading, blots were stripped and hybridised with a probe spanning the coding region of the Arabidopsis glyceraldehyde-3P-dehydrogenase cytosolic gene $(\mathrm{GapC})$. For RT-PCR analysis cDNA synthesis was performed on 1 $\mu \mathrm{g}$ total RNA (DNase treated) using oligo-dT(15) primer. The volume of these cDNA mixtures was increased to $100 \mu \mathrm{l}$ and for each PCR reaction $5 \mu \mathrm{l}$ of the cDNA mixtures (equivalent to $0.05 \mu \mathrm{g}$ RNA) was added to $15 \mu$ PCR mix. Expression of mac12.6 was detected using the RTL2 primers amplifying a $322 \mathrm{bp}$ fragment from cDNA and a 396 bp fragment from genomic DNA. Expression of $G a p C$ was detected using the GapC primers, which amplify a $530 \mathrm{bp}$ fragment from cDNA and a $1,300 \mathrm{bp}$ fragment from genomic DNA. Semiquantitative PCR amplification was performed at 20 cycles. The PCR products were detected by DIG-labelled probes after separation on an agarose gel and Southern blotting. Expression of LEP was detected by the RTL1 primers, which amplify a 254 bp fragment 3 ' of the DNA binding domain of $L E P$. The pLEP primers and combinations of these pLEP primers with the primers 35SleaderF and RTL1R identified the 35 S transcript. PCR amplification was performed for 35 cycles and the resulting PCR products were separated on an agarose gel. Autoradiograms of the blots and gel images were scanned and processed using the Adobe PhotoShop computer program.

\section{RACE PCR}

The SMART RACE cDNA amplification kit (CLONTECH) was used for 5' and 3' RACE PCR with the LEP5'race, LEP3'race and PROM5'race primers for analysis of the LEP transcription start and polyadenylation site and the transcription start site of the $35 \mathrm{~S}$ transcript, respectively. The resulting PCR products were cloned in the pGEM-T-easy vector (Promega) and at least 10 independent clones were sequenced for each analysis.

\section{Anatomical analysis}

Plant material was fixed in $2 \%$ glutaraldehyde in $0.1 \mathrm{M}$ sodium cacodylate buffer ( $\mathrm{pH}$ 7.2) for 8 hours at room temperature (RT), dehydrated through an ethanol series and embedded in Epon. Sections $(1 \mu \mathrm{m})$ were stained with Toluidine Blue and mounted in Epon.

\section{SEM analysis}

Plants were grown in tissue culture and fixed for 2 days in FAA $(50 \%$ ethanol, $5 \%$ acetic acid and $3.7 \%$ formaldehyde). The material was washed in $70 \%$ acetone and further processed as described by Schneitz et al. (1997).

\section{In situ hybridisation with DIG-labelled RNA probes}

Part of the LEP coding region excluding the AP2/EREBP domain was amplified by PCR using the RTL1 primers. The resulting PCR fragment was cloned in the pGEM-T-easy vector (Promega) and DIGlabelled antisense and sense probes were synthesised using the DIG RNA labelling kit (Boehringer) from linearised vectors. Plant material was fixed in FAA for 10-12 hours, dehydrated in a graded ethanol series and embedded in Steedman's wax. Sections ( $8 \mu \mathrm{m}$ thick) were cut and mounted on polylysine-coated slides (Sigma), which were baked overnight in a $30^{\circ} \mathrm{C}$ oven. In situ hybridisation analysis was performed essentially as described by Vielle-Calzada et al. (1999) with minor modifications: Proteinase $\mathrm{K}(1 \mu \mathrm{g} / \mathrm{ml})$ was used instead of Pronase $\mathrm{E}$ and the postfixation was followed by treatment with acetic anhydride for 10 minutes. The colour reaction was performed using Western Blue (Promega) as substrate (including 7.6 mM levamisole). The slides were stained overnight, washed in TE, mounted in glycerol:TE (1:1) and photographed using Nomarski optics.

\section{RESULTS}

\section{Altered development of the let mutant}

In a screen for leaf developmental mutants we isolated a transgenic line exhibiting a dominant leaf phenotype. Because of its conspicuous leafy petiole phenotype, which consisted of the formation of leaves without a petiole (Fig. 2A-D), this mutant was called lettuce (let). The total width and length of 
Fig. 1. Schematic representation of the T-DNA insert with flanking plant DNA isolated from let and the derived T-DNA constructs. (A) Using HindIII and Pst I, $2.9 \mathrm{~kb}$ and $11.3 \mathrm{~kb}$ of plant DNA flanking the T-DNA insert was isolated via plasmid rescue. The PstI fragment was subcloned into 5 smaller fragments to generate probes for northern blot analysis using the indicated HindIII and XhoI sites. Part of the $2.9 \mathrm{~kb}$ HindIII fragment directly flanking the T-DNA insert is shown in detail. On this fragment two genes are located: LEP (41057-41689) and mac12.6 (42226-42794). LEP contains a 93 bp 5'UTR (40964-41056) and a 197 bp 3'UTR (41690-41886). Numbers indicate the location on the BAC clone MAC12. The numbered arrowheads indicate the position of the primers used in this study, as follows. $(1,2)$ : RTL1F and RTL1R, $(3,4)$ : RTL2F and RTL2R, $(5,6,7)$ : pLEPF, pLEPR and 35SleaderF and $(8,9,10)$ : LEP5'race, LEP3'race and prom5'race. Stippled box: 5'UTR and 3'UTR region of $L E P$. Broken line: pUC9 sequences present in the activator T-DNA used for plasmid rescue. (B) The T-DNA constructs derived from the $2.9 \mathrm{~kb}$ HindIII fragment directly flanking the RB (shown in A). This HindIII fragment (35SDE-LEP-mac12.6) was used to generate the $35 \mathrm{SDE}-L E P$ construct upon removal of the mac12.6 coding region using the $A c c$ I restriction site at position 42174 just upstream of the mac12.6 coding region. DE-LEP was obtained by omitting the $35 \mathrm{~S}$ minimal promoter region and AMV leader sequence (black box) from 35SDE-LEP. Open boxes: $L E P$ and mac12 coding region. Black box: $35 \mathrm{~S}$ minimal promoter ( -90 to 0$)+$ AMV leader sequence. Hatched box: $35 \mathrm{~S}$ enhancer region ( -393 to -95$)$. Arrows indicate the different $L E P$ transcripts, which are generated by the T-DNA constructs

the mutant leaves were comparable to that of wild type (Fig. 2C,D). The largest rosette leaf on wild-type plants was on average $23 \mathrm{~mm}$ long and $9 \mathrm{~mm}$ wide with the petiole comprising $29 \%$ of total leaf length $(n=29)$, while for let the dimensions for the largest rosette leaf were $25 \mathrm{~mm}$ long and 9 $\mathrm{mm}$ wide without petiole $(n=15)$. The penetrance of the leafy petiole phenotype was not complete in the first leaf pair (Fig. $2 \mathrm{R})$. In let the petiole constituted $25 \%$ and $24 \%$ of total leaf length for the first and second rosette leaf $(n=15)$ respectively, while all subsequently formed leaves lacked a petiole. In wild-type plants, petiole length was $45 \%$ and $43 \%$ of total leaf length for the first and second rosette leaves $(n=29)$ respectively, with petiole length gradually decreasing to $4 \%$ of total leaf length for the last rosette leaf formed. Leaf blade anatomy of wild-type and mutants leaves was studied. This showed that the size and shape of the cells in the mutant leaves, including the leafy petioles, were comparable to those in wildtype leaves in both longitudinal and cross sections (Fig. 3). Therefore, the lack of a petiole in the mutant leaves was not caused by altered cell elongation but was the result of the conversion of the proximal petiole into a leaf blade.

Closer examination of leaf formation using SEM analysis showed that the earliest difference that could be observed between wild type and let was in the primary leaves of plants 6 days after germination. At this stage wild-type leaves started to form a petiole (Fig. 2I). No significant petiole was formed on let leaves at the same developmental stage, while the leaf blade was larger (Fig, 2J). No difference could be observed in the morphology of the apex, the leaf primordia or the primary leaves shortly after the outgrowth of the leaf primordia (data not shown). Leaf initiation in let (Fig. 2O-R) followed the same phyllotaxis as wild type (Fig. 2K-N). Besides altered leaf development, inflorescence branching of let was aberrant (Fig. $2 \mathrm{E}, \mathrm{F}$ ) and the mutant carpels/siliques were short and thick (Fig. $2 \mathrm{G}, \mathrm{H}$ ) resembling those formed by the erecta mutant (Torii et al., 1996). Cotyledon development was comparable with that of wild-type plants. Although petioles are not formed on cauline leaves, the proximal part of the let cauline leaves was wider than those of wild type and resembled mutant rosette leaves in shape. Except for the alteration in carpel/silique shape, the mutant flower organs were identical to wild type (data not shown).

\section{Genetic analysis of the let mutant}

The T-DNA locus closely linked with the let mutant phenotype (see Materials and Methods) and the plant DNA flanking the $\mathrm{RB}$ were isolated by plasmid rescue (Fig. 1A). Subfragments of the $11.3 \mathrm{~kb}$ flanking plant DNA isolated using Pst (Fig. 1A) were used as probes in northern blot analysis. Only with the $2.9 \mathrm{~kb}$ fragment directly flanking the RB of the T-DNA insert as probe was a difference in gene expression detected between wild type and let (data not shown). Sequence analysis of the isolated plant DNA and comparison with entries in DNA databases revealed that the isolated DNA showed $100 \%$ sequence identity to a region located on the BAC clone MAC12 and, therefore, the T-DNA had inserted on the top (4.6 Mb) of 
Fig. 2. The let mutant exhibits an altered leaf, inflorescence and silique formation. Development of wild-type (A,C,E,G,I,K-N) and let (B,D,F,H,J,O-R) plants. (A-D) 3week-old seedlings; (A,B) whole plant, $(\mathrm{C}, \mathrm{D})$ leaves, $(\mathrm{E}, \mathrm{F})$ inflorescence, $(\mathrm{G}, \mathrm{H})$ matured siliques. (I,J) SEM images of primary leaves taken from 6-day-old plants. (K-N and O-R)

Developmental series of one wildtype and one let plant, respectively. Pictures were taken $7(\mathrm{~K}, \mathrm{O}), 9$ (L,P), $11(\mathrm{M}, \mathrm{Q})$ and $13(\mathrm{~N}, \mathrm{R})$ days after germination. Scale bars, $1 \mathrm{~cm}$ (A-F), $100 \mu \mathrm{m}(\mathrm{I}, \mathrm{J})$ and $0.32 \mathrm{~cm}(\mathrm{~K}-$ R). c: cotyledons.
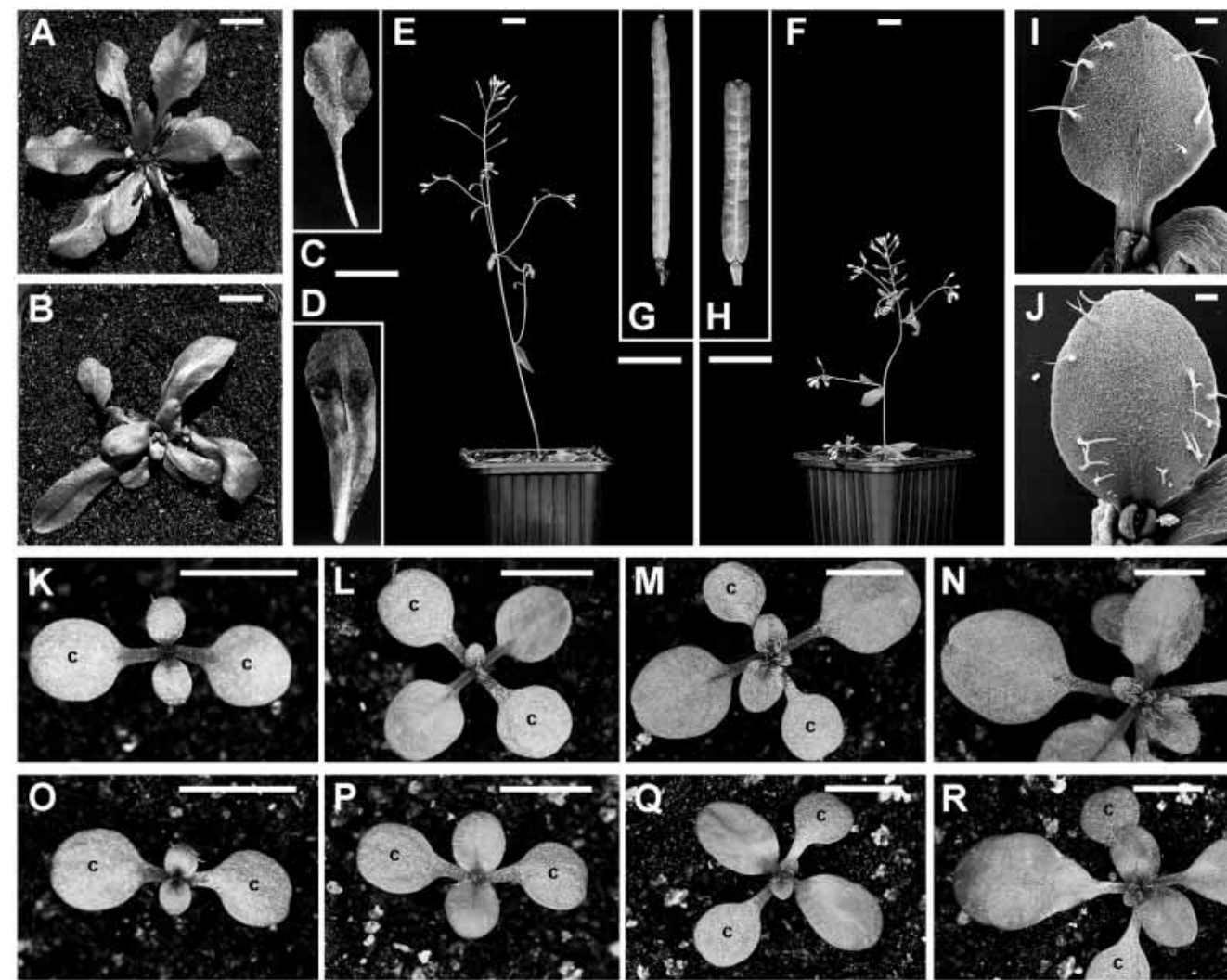

chromosome $\mathrm{V}$ represented by position 40.351 of MAC12 (Sato et al., 1997). Two genes were located on the $2.9 \mathrm{~kb}$ fragment directly flanking the RB of the T-DNA insert (Fig. 1A). The T-DNA causing the mutant phenotype inserted 706 bp upstream of the ATG start codon of mac12.5, which we call $\underline{L E A F Y} \underline{P E T I O L E}$ (LEP; GenBank AF216581) and 1915 bp upstream of the ATG start codon of mac12.6. Southern blot analysis showed that both LEP and mac12.6 are single copy
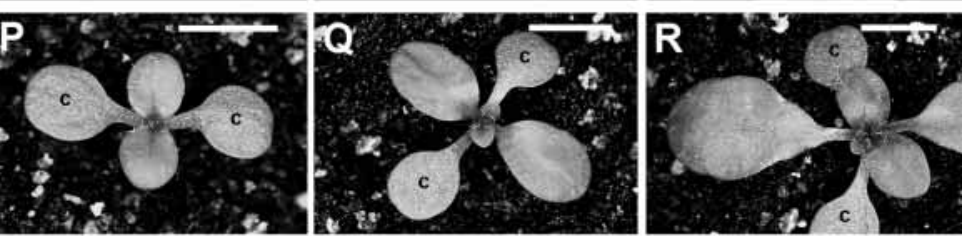
genes (data not shown).

\section{LEP is a member of the AP2/EREBP family}

No significant similarity with known genes was previously detected for LEP (Sato et al., 1997). However, when we inspected the DNA sequence more carefully, we found that $L E P$ encodes a protein that is hydrophilic and contains a region of 57 amino acids which shares a significant similarity with the DNA binding domain of the AP2/EREBP family of plant transcription factors (Weigel, 1995; Riechmann and Meyerowitz, 1998). The protein contains a putative nuclear localisation signal near the $\mathrm{N}$ terminus and a proline-rich region between

Fig. 3. Anatomy of wild-type and let leaf blade. Leaves were isolated from 2-week-old plants grown in soil and sections (A,B, cross; C,D, longitudinal) were made at different positions in the leaf blades.

Representative sections are shown for $(\mathrm{A}, \mathrm{C})$ wild type, $(\mathrm{B}, \mathrm{D})$ let. All pictures are shown at the same magnification.

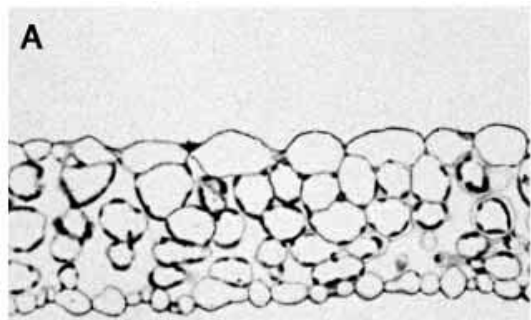

B

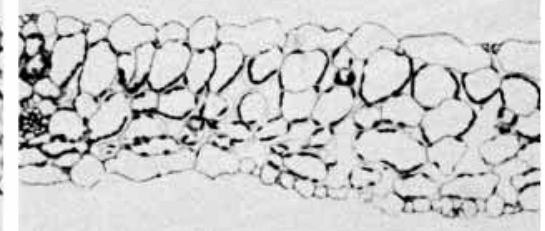

C domain. Therefore, $L E P$ is likely to encode a plant transcription factor. The LEP protein shares the highest similarity with EREBP-3, a member of the tobacco ethylene responsive element binding proteins (Ohme-Takagi and Shinshi, 1995) and as in EREBP-3 the AP2/EREBP domain of LEP is located close to the N-terminus of the protein. The LEP proline-rich region (aa 81-105) showed a high score in 
the PESTfind analysis. Such PEST sequences, which are enriched for proline $(\mathrm{P})$, glutamine $(\mathrm{E})$, serine $(\mathrm{S})$ and threonine $(\mathrm{T})$, were found to be responsible for rapid proteolytic degradation (Rogers et al., 1986; Rechsteiner and Rogers, 1996). Examination of a subset of genes belonging to the AP2/EREBP family showed that EREBP1-3, DREB2A, AP2 and RAP2.4 also gave significant scores for the presence of PEST sequences in this PESTfind analysis. On the other hand, no significant scores were obtained for EREBP4, CBF, ANT, TINY, AtEBP and the other RAP and DREB proteins. This suggests that rapid proteolytic degradation could be a feature shared by a subset of genes belonging to the AP2/EREBP family.

\section{Altered expression of $L E P$ and mac12.6 caused by activation tagging}

Northern blot analysis showed that LEP was very weakly expressed in wild type and could only be detected in young shoots. Wild-type LEP expression was most obvious in 1week-old plantlets and to a lesser extent in 2-week-old shoots (data not shown). LEP expression was significantly increased in let compared to wild type (Fig. 4A). Two transcripts were detected in let with a probe for LEP. The smaller transcript migrated the same distance (approximately $900 \mathrm{bp}$ ) as the transcript in wild type (Fig. 4A, lane 2) and, therefore, probably originated from transcription initiated on its own promoter, which is likely to be enhanced by the activator tag. The larger transcript was approximately 1800 bp long. Using RACE PCR the position of the putative transcription start and polyadenylation site in $L E P$ was determined (Fig. 1A). This showed that LEP contains a 93 bp 5'UTR and 197 bp 3'UTR, resulting in a $923 \mathrm{bp}$ transcript without the polyA-tail. Using different primer combinations (35SleaderF, pLEPF, pLEPR and RTL1R; Fig. 1A) the larger transcript was shown to result from transcription initiation from the $35 \mathrm{~S}$ CaMV promoter, used as activator tag. The start of transcription in the activator tag was confirmed by RACE PCR. The size of this $35 \mathrm{~S}$ transcript was shown to be $1605 \mathrm{bp}$ without the polyA-tail. None of the hypothetical proteins that could be translated from this $35 \mathrm{~S}$ transcript, besides the LEP protein, was longer than 25 amino acids. Thus, it is unlikely that such a protein would have a function. Wild-type expression of mac12.6 and even the increased expression levels in let could not be convincingly detected in northern blot experiments (data not shown). Therefore, semi-quantitative RT-PCR was performed to study mac12.6 expression. This showed that mac12.6 expression was increased approximately 10 - to 20 -fold in let compared to wild type (Fig. 4B).

\section{Activation tagging of LEP causes altered leaf, inflorescence and silique development}

To study the role of LEP and mac12.6 in the establishment of the altered development of let, the 35SDE-LEP-mac12.6 and 35SDE-LEP T-DNA constructs (Fig. 1B) were transformed into wild-type plants. After the transformation of these T-DNA constructs the let phenotype (leafy petioles, aberrant inflorescence branching and altered carpel/silique shape) was observed among both sets of primary transformants. The analysis of the progenies derived from the primary transformants showed that most of the transgenic lines exhibited the leafy petiole phenotype, albeit to a varying degree. Among the 16 35SDE-LEP-mac12.6 transgenics, 3 lines exhibited a wild-type development, 3 lines a weak, 3 lines an intermediate and 7 lines the strong (comparable to the original let mutant) leafy petiole phenotype, while among the 31 35SDE-LEP transgenics, 5 lines exhibited a wild-type, 5 lines a weak, 8 lines an intermediate and 13 lines the strong leafy petiole phenotype. $L E P$ expression was analysed by northern blot analysis for 12 transgenics (35SDE-LEPmac12.6 and 35SDE- $L E P$ ) exhibiting the leafy petiole phenotype and 3 transgenics displaying a wild-type development. This analysis showed that an increased LEP expression could only be detected in the 12 transgenics exhibiting the mutant phenotype. For both the 35SDE-LEPmac12.6 and 35SDE-LEP transgenics the two transcripts detected in let were also observed. No correlation between LEP expression levels and strength of the leafy petiole phenotype was observed (Fig. 4D). Because the establishment of the let mutant phenotype was observed in both the 35SDE-LEPmac12.6 and 35SDE-LEP transgenics and only in those transgenics exhibiting an increased LEP expression, we conclude that the altered development of let was caused by activation tagging of the $L E P$ gene.
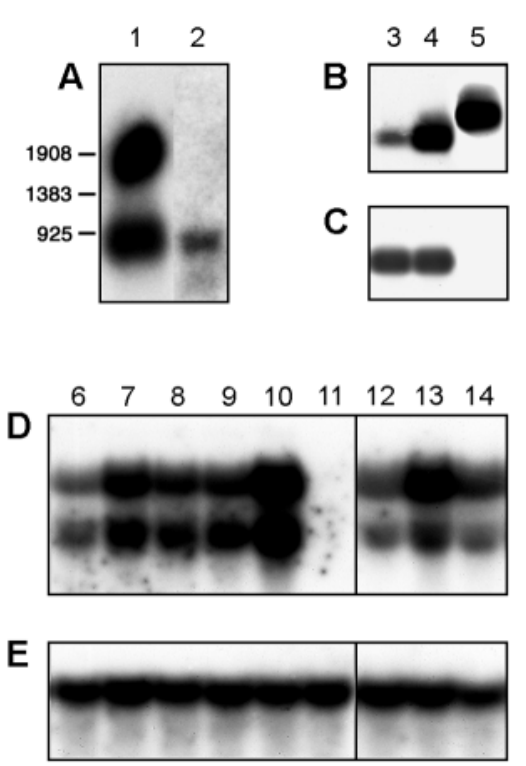

Fig. 4. Two LEP transcripts can be detected in let and 35SDE- LEP overexpressors. (A,D) Northern blot analysis of $L E P$ expression. $(\mathrm{B}, \mathrm{C})$ semi-quantitative RT-PCR of mac12.6 and $\mathrm{GapC}$ expression, respectively. (E) GapC loading control. (A) Lane 1: $1 \mu \mathrm{g}$ poly(A) ${ }^{+}$ RNA from 2-week-old let mutant, lane $2: 2 \mu \mathrm{g}$ poly $(\mathrm{A})^{+} \mathrm{RNA}$ from 2 -week-old wild type. The filter was hybridised with a probe for LEP. The expression shown in lane 1 was obtained after 16 hours and the expression shown in lane 2 after 5 days of exposure. $(\mathrm{B}, \mathrm{C})$ Lanes 3 and 4: PCR on cDNA (equivalent to $0.05 \mu \mathrm{g}$ RNA) from wild type and let, respectively. Lane 5: PCR on $0.05 \mu \mathrm{g}$ genomic DNA from wild type, showing that none of the cDNA samples (lanes 3 and 4) contained a detectable genomic DNA contamination. (D,E) Lanes 6-11: 35SDE-LEP-mac12.6 transgenics exhibiting a weak (6), intermediate (7 and 8), strong leafy petiole phenotype (9 and 10) and a wild-type development (11). Lanes 1214: 35SDE-LEP transgenics exhibiting a strong (12), intermediate (13) and weak leafy petiole phenotype (14). Total RNA $(20 \mu \mathrm{g})$ was loaded for lanes 6-14. 
Fig. 5. $L E P$ is ectopically expressed in most organs of let and $35 \mathrm{SDE}-L E P$ overexpressors. PCR was performed on cDNA (from $0.05 \mu \mathrm{g}$ RNA) isolated from cotyledons (cotyl), old non-expanding leaves (leaf), inflorescence-stem (infl) and flowers taken from wildtype plants (C24), let and a 35SDE-LEP transgenic (35S-LEP) exhibiting a strong leafy petiole phenotype. As positive controls, PCR was performed on $0.05 \mu \mathrm{g}$ genomic DNA from wild type or 5 ng vector DNA (containing the $2.9 \mathrm{~kb}$ HindIII fragment; see Fig. 1A). LEP expression (LEP coding) was detected using the RTL1 primers and amplification from the individual cDNA samples was verified with GapC primers. PCR using the GapC primers (-) was performed on $0.05 \mu \mathrm{g}$ RNA as a control for genomic DNA contamination in the different samples.

\section{LEP is expressed in leaf primordia and developing leaf blades}

To characterise wild-type $L E P$ expression in more detail we studied $L E P$ expression in those organs that were developmentally affected in let and the 35SDE-LEP overexpressors (i.e. the 35SDE-LEP-mac12.6 and 35SDE-LEP transgenics displaying the strong leafy petiole phenotype). Because of the low wild-type $L E P$ expression levels further analysis of $L E P$ expression was performed using RT-PCR analysis (Fig. 5). In wild type no LEP expression could be detected in the cotyledons, non-expanding (old) leaves,

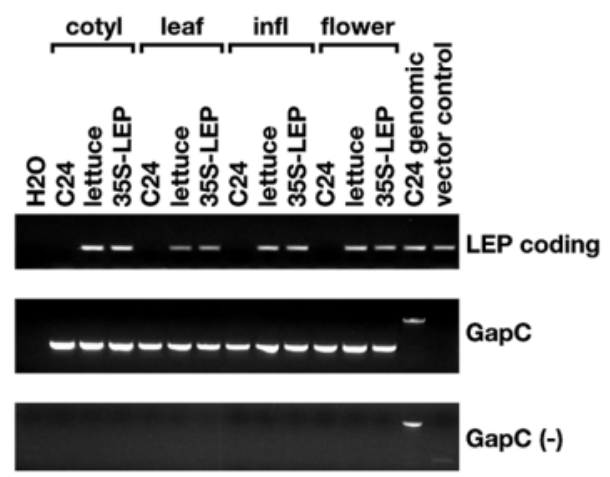

inflorescence stems or flowers. Therefore, LEP is only expressed in young shoots of wild-type plants. In let and the $35 \mathrm{SDE}-L E P$ overexpressors $L E P$ expression was detected in all organs analysed.

Because wild-type $L E P$ expression appeared to be restricted to young shoots, the expression of $L E P$ was analysed at this developmental stage in more detail with in situ RNA hybridisation (Fig. 6). LEP expression patterns at this developmental stage were similar in wild type, let and 35SDE$L E P$ overexpressors (Fig. 6B). In wild type, $L E P$ was expressed throughout very young leaf primordia just emerging from the

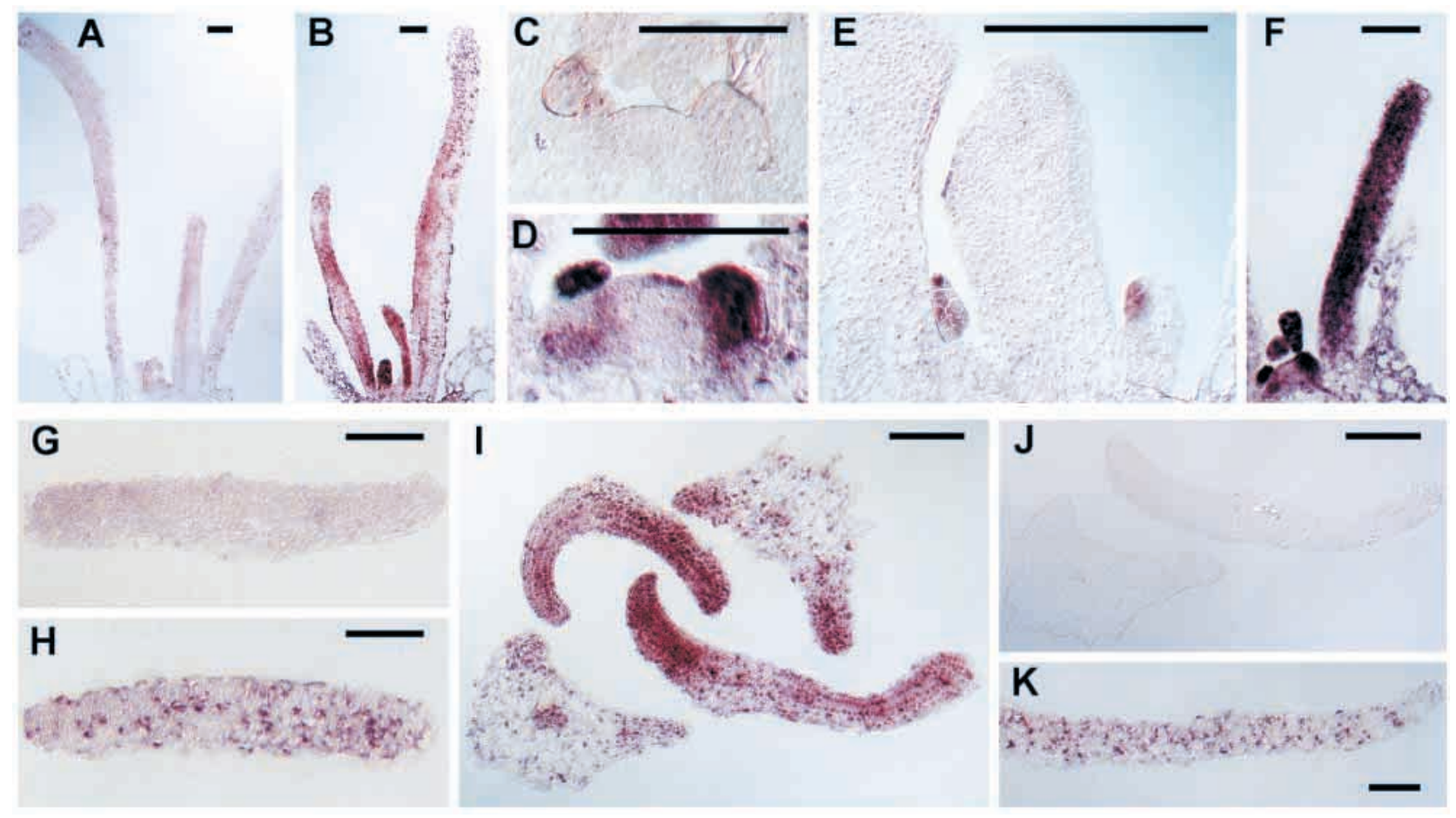

Fig. 6. $L E P$ shows similar expression in young shoots of wild type, let and 35SDE- LEP overexpressors. (A-F) Longitudinal sections through 7day-old wild-type shoots. (A) No signal was detected with the $L E P$ sense probe. (B) Representative staining pattern obtained with the $L E P$ antisense probe in wild type, let and 35SDE-LEP overexpressors. Staining was found throughout the whole leaf primordia with strongest expression in the youngest primordium, gradually decreasing in subsequent older primordia. (C,D) Detail showing the shoot apical meristem region and the emerging leaf primordium hybridised with the $L E P$ sense (C) and antisense (D) probe, respectively. (E) Expression in stipules could be detected prior to expression in leaf primordia. (F) Detail of leaf primordium showing strong signal throughout the whole primordium after more prolonged development of the colour reaction than in E. (G-K) Cross sections through 7-day-old wild-type shoots showing leaves at different developmental stages. $(\mathrm{G}, \mathrm{H})$ Young developing leaves hybridised with the $L E P$ sense $(\mathrm{G})$ and antisense probe $(\mathrm{H})$. (I,J) Young leaves shown at different stages of development hybridised with the $L E P$ antisense (I) and sense probe (J). Note the stronger $L E P$ expression in the young developing leaf blade compared to the more mature leaf blade and the midrib in I. (K) Older more expanded leaf blade hybridised with the $L E P$ antisense probe. Scale bars, $100 \mu \mathrm{m}$. 

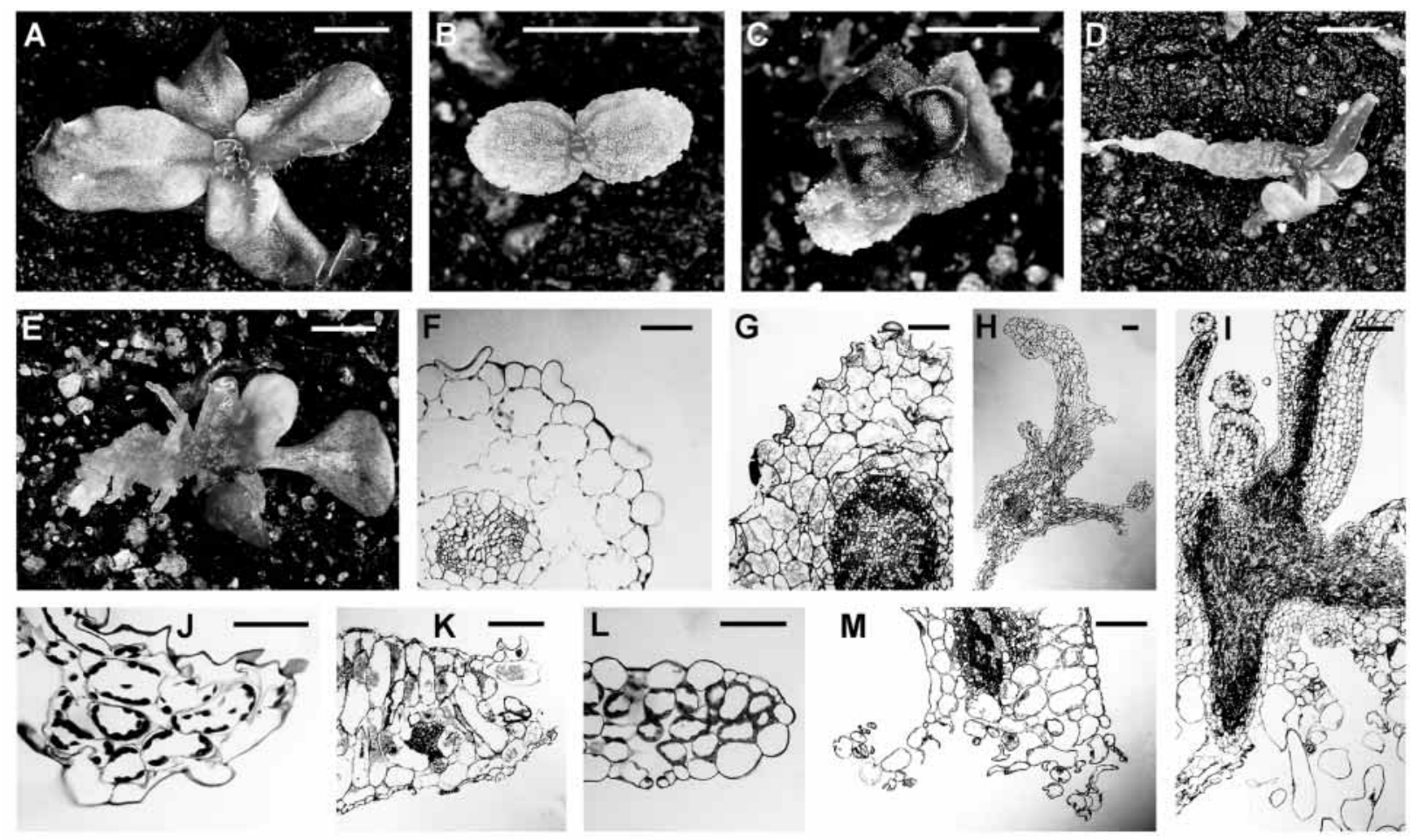

Fig. 7. Development of DE-LEP and cDNAoe transgenics. (A) Two-week-old DE- LEP transgenic plant exhibiting a strong leafy petiole phenotype. (B-E) Two- to 4-week-old cDNAoe transgenics. (B) cDNAoe-A, (C) cDNAoe-B and (D,E) cDNAoe-C transgenics producing a longer and thicker hypocotyl with callus like appearance (D) and ectopic outgrowths $(\mathrm{E})$. (F-H) Cross section through hypocotyls from wild type $(\mathrm{F})$, and $(\mathrm{G})$ cDNAoe-A and $(\mathrm{H})$ cDNAoe-C transgenics. Note the increase in the number of cell layers in $(\mathrm{G})$ compared to $(\mathrm{F})$ and the ectopic outgrowths in (H). (I) Longitudinal section through a cDNAoe-B shoot. Note the loss of tissue organisation at the base of the hypocotyl and the increase in vascular tissue. $(\mathrm{J}, \mathrm{K})$ Cross sections through cotyledons from wild type $(\mathrm{J})$ and cDNAoe-A or cDNAoe-B transgenic (K). $(\mathrm{L}, \mathrm{M})$ Cross sections through leaves from wild type (L) and cDNAoe-B transgenic (M). Note the gap at the blade edge in both the cotyledons and leaves from the transgenics (K,M) compared to wild type (J,L). Scale bar, $0.2 \mathrm{~mm}$ in (A-E) and $100 \mu \mathrm{m}$ in (F-M).

shoot apical meristem (Fig. 6D). Short development of the colour reaction detected LEP expression in stipules before expression in leaf primordia could be detected (Fig. 6E), indicating stronger $L E P$ expression in stipules compared to leaf primordia. This result was corroborated by analysis of transgenics harbouring a LEP promoter (-1670)-GUS fusion construct. In less than $10 \%$ of the primary transformants harbouring this promoter-GUS fusion GUS activity was detected in the shoot where it was restricted to the stipules. No GUS activity could be observed in the primordia or young leaves of such lines (data not shown). Longer development of the colour reaction compared to the staining in Fig. 6E revealed staining in all cells constituting the young leaf primordia (Fig. 6F). LEP expression could also be detected in young developing leaves where it appeared to be restricted to the leaf blades (Fig. 6H,I). LEP expression was strongest in very young leaf blades and became weaker upon further expansion of these blades (Fig. 6I,K). In vacuolated cells containing chloroplasts background staining caused by the chloroplasts interfered with reliable detection of $L E P$ expression, thus preventing analysis of leaves at older stages (data not shown). LEP expression patterns in let and the 35SDE-LEP overexpressors were shown to be identical to those in wild type and, therefore, we conclude that $L E P$ expression was increased in a tissue-specific way in those organs where wild-type $L E P$ expression could be detected.

\section{The 35S transcript initiated in the activator tag is not involved in the establishment of the mutant phenotype}

To study the role of the $35 \mathrm{~S}$ transcript $(1800 \mathrm{bp})$ in the establishment of the mutant phenotype, the DE-LEP construct was made (Fig. 1B) in which the $35 \mathrm{~S}$ minimal promoter was removed in comparison to the $35 \mathrm{SDE}-L E P$ construct. Primary transformants and their progeny harbouring this DE-LEP construct exhibited the leafy petiole phenotype (Fig. 7A), but also the mutant inflorescence and silique/carpel phenotype. Similar to the observation for the 35SDE-LEP overexpressors the individual DE-LEP lines displayed these phenotypes at different strengths. PCR analysis confirmed that the $35 \mathrm{~S}$ minimal promoter region was absent in these transgenics (data not shown), while northern blot analysis proved that in the DELEP lines exhibiting the leafy petiole phenotype only the 900 bp transcript was present (Fig. 8A, lanes 2-4). Because the DE$L E P$ transgenics displayed exactly the same phenotype as let and the 35SDE-LEP transgenics, we conclude that the $35 \mathrm{~S}$ transcript does not contribute to the establishment of the mutant phenotype.

\section{Constitutive overexpression of LEP causes increased and ectopic cell divisions}

To study the effect of constitutive overexpression of $L E P$ on plant development, the coding region of $L E P$ was amplified by 


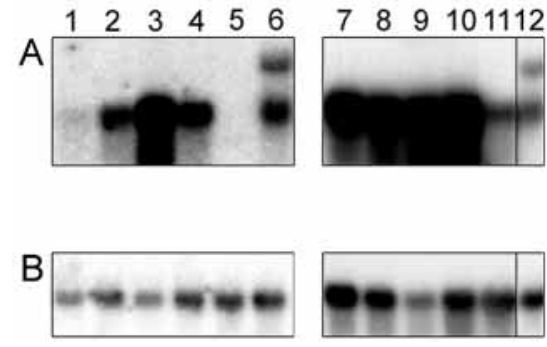

Fig. 8. $L E P$ expression in the DE- $L E P$ and cDNAoe transgenics. (A) Northern blot analysis of $L E P$ expression and (B) GapC loading control. Lanes 1-4: four independent primary transformants harbouring the DE-LEP construct with (1) wild-type phenotype and (2-4) exhibiting an increase in the strength of the leafy petiole phenotype; lane 5: wild type; lanes 6 and 12: 35SDE-LEP transgenic exhibiting a strong leafy petiole phenotype; lanes 7-11: cDNAoe transgenics with (7) old and (8) young cDNAoe-B transgenics, (9) young and (10) old cDNAoe-A transgenics, and (11) cDNAoe-C transgenics. Lane 12 shows a fourfold longer exposure for the $L E P$ probe compared to lanes $7-11$. In each case $5 \mu \mathrm{g}$ total RNA ( $2 \mu \mathrm{g}$ for lanes 1, 3 and 9) isolated from plants grown in tissue culture was loaded. For the DE- LEP transgenics RNA was isolated from single plants, while for the cDNAoe transgenics RNA was isolated from 1020 pooled transgenics exhibiting a similar phenotype.

PCR and cloned in a $35 \mathrm{~S}$ overexpression cassette. The primary transformants harbouring the resulting cDNAoe construct had severe developmental defects (Fig. 7B-M). Three main classes of plants exhibiting distinct altered phenotypes (cDNAoe-A, -B and $-\mathrm{C}$ ) could be observed. The cDNAoe-A transformants were most severely affected, developed only cotyledons and occasionally very small primary leaves (Fig. 7B). The hypocotyl of such plants was swollen at the base and the edge of the cotyledons was rough rather than smooth (Fig. 7B). The cDNAoe-B plants formed several rosette leaves but they remained small and the plants looked dwarfed (Fig. 7C). In older plants the leaf and cotyledon edge looked rough, and here also, the base of the hypocotyl was swollen. The cDNAoe-C plants formed a minor group and exhibited a longer hypocotyl. A subset of these cDNAoe-C plants had normal or slightly distorted rosette leaf formation. But in all cases the hypocotyl was thicker, with the surface having a callus like appearance (Fig. 7D). In the more severe cases, the hypocotyl had ectopic outgrowths (Fig. 7E). Anatomical analysis of cDNAoe transgenics revealed increased (Fig. 7G) and/or ectopic cell divisions (Fig. 7H). More cell layers were observed in the vascular tissue and cortex of the hypocotyl, and vascular tissue formation was also increased in leaves and cotyledons (Fig. 7I). Leaves produced by cDNAoe-B plants initially showed a wildtype morphology, but upon further development exhibited gaps at the leaf edge resulting in a rough appearance. The formation of the rough cotyledon and leaf edges on cDNAoe-A and -B plants was caused by a loss of connection between ventral and dorsal blade tissue (Fig. 7K,M). Anatomical analysis showed that there is a complete loss of cell organisation in the swollen hypocotyl base (Fig. 7I). Only the vascular tissue seems to remain intact. It proved impossible to obtain seeds from the cDNAoe transgenics since these plants died before bolting when grown in both tissue culture and soil.

Northern blot analysis (Fig. 8A, lanes 7-12) indicated that
LEP was highly overexpressed in the primary transgenics with highest expression in the cDNAoe-A and -B transgenics, which were most severely affected in development. Compared to a $35 \mathrm{SDE}-L E P$ overexpression line exhibiting the strong leafy petiole phenotype, $L E P$ expression was 20 - to 50 -fold stronger in those cDNAoe transgenics.

\section{DISCUSSION}

\section{LEP is a member of the AP2/EREBP family of transcription factors}

LEP encodes a putative transcription factor, which shares sequence similarity with the DNA binding domain of members of the AP2/EREBP family of transcription factors (Weigel, 1995; Okamuro et al., 1997; Riechmann and Meyerowitz, 1998). The number of genes constituting this family in Arabidopsis has been estimated to be about 125 (Riechmann and Meyerowitz, 1998). The family can be divided into two subfamilies. The EREBP subfamily members contain one DNA-binding domain, while the AP2 subfamily members contain two DNA-binding domains, which may permit these proteins to bind to DNA as a single polypeptide (Moose and Sisco, 1996). In LEP, only one such element is present and because of this may require homo/heterodimer formation for DNA binding activity. The functionally characterised proteins containing two DNA-binding domains are known to be involved in the control of development, whereas those harbouring one DNA-binding domain, with the exception of TINY (Wilson et al., 1996), are involved in stress-related responses. Apparently, LEP is the second protein containing one DNA-binding domain, which is involved in development. It may not be coincidental that $L E P$, like TINY, was isolated by activation tagging. $L E P$ shares the highest similarity with $E R E B P 3$, a tobacco gene involved in ethylene-induced pathogen defence. However, activation tagging of $L E P$ in Arabidopsis caused a leafy petiole phenotype as a result of ectopic leaf blade formation, and thus LEP could be involved in leaf blade development.

\section{Altered LEP expression caused by activation tagging}

In let and 35SDE-LEP overexpressors, two LEP transcripts (1800 and $900 \mathrm{bp}$, respectively) were identified by northern blot analysis, but in DE-LEP plants only the smaller of these two was present. Since all the phenotypes associated with the let mutant were also seen in the DE-LEP transgenics, the larger $35 \mathrm{~S}$ transcript apparently does not contribute to the phenotype. Translation of the long transcript, which has a number of small ORFs in front of the LEP coding sequence, may not generate LEP protein, explaining the lack of contribution of this $35 \mathrm{~S}$ transcript to the phenotype. The shorter ( $900 \mathrm{bp}$ ) transcript was upregulated in let and both the 35SDE-LEP and DE-LEP transgenics showing an altered development. In situ hybridisation analysis showed that the pattern of $L E P$ expression in the young shoot was similar in wild type, let and the 35SDE-LEP overexpressors. Apparently, the activator tag acts as an enhancer of the $L E P$ promoter causing tissue-specific upregulation of $L E P$ expression. However, by RT-PCR analysis additional ectopic $L E P$ expression was detected in the overexpressors. 


\section{Altered leaf development caused by activation tagging of $L E P$}

The analysis of wild-type and mutant leaves showed that the leafy petiole phenotype was not caused by a defect in cell expansion. Instead, activation tagging of $L E P$ resulted in conversion of the proximal petiole into an ectopic leaf blade; i.e. the leaf blade invaded the petiole region. This suggests that LEP might be a positive regulator of leaf blade formation. LEP activity could provoke leaf blade formation if LEP was required for the establishment of distal domain identity, thereby functioning in proximodistal patterning. Alternatively, LEP could be involved in cell division activity in the marginal meristem. The establishment of the leafy petiole phenotype caused by activation tagging of $L E P$ could have been brought about by several mechanisms. In situ hybridisation analysis detected wild-type $L E P$ expression throughout the whole leaf primordia. Therefore, it is likely that the activity of LEP in leaf primordia is regulated at the protein rather than the transcriptional level similar to the proposed regulation of PHAN activity (Waites et al., 1998). LEP activity could be suppressed in the proximal domain by either a negative regulator or by increased degradation of the LEP protein in this part of the leaf primordia. LEP could also interact with other transcription factors encoded by genes exhibiting expression specific for the distal region. Activation tagging of $L E P$ might result in higher LEP protein levels in the proximal domain of leaf primordia, which might be too high to be completely inactivated or degraded. Alternatively, higher LEP protein levels could lead to either homodimer formation or heterodimer formation with partners that LEP normally does not interact with. Individual lines harbouring either the 35SDELEP-mac12.6, 35SDE-LEP or DE-LEP constructs displayed the leafy petiole phenotype at different strengths rather than an all-or-nothing conversion of petiole to leaf blade suggesting that LEP protein levels are related to the degree of leaf blade invasion into the petiole. This can be explained if LEP protein activity needs to reach a certain threshold level for stimulation of leaf blade formation.

Wild-type $L E P$ expression is restricted to leaf primordia, stipules and developing leaf blades. This expression pattern of $L E P$ resembles that of the cyclin cyclAt promoter used to study cell division activity during Arabidopsis leaf development (Donnelly et al., 1999). That study showed that initially, leaf blade formation is correlated with localised cell division at the primordium margin, while subsequent growth of the tissue layers is established by a more diffuse pattern of cell division in the developing leaf blade. Furthermore, high constitutive $L E P$ expression displayed by the cDNAoe transgenics causes increased and ectopic cell divisions. Therefore, $L E P$ is likely to act as a positive regulator of cell division activity in both the marginal meristem and during the subsequent establishment of the tissue layers making up the leaf blade.

\section{Altered development caused by ectopic LEP expression}

Activation tagging of $L E P$ not only causes the leafy petiole phenotype but also affects inflorescence and carpel/silique development. High constitutive $L E P$ overexpression in the cDNAoe transgenics results in increased and ectopic cell divisions and loss of cell integrity in several organs. It is unclear how ectopic $L E P$ expression can result in such diverse alterations in plant development. Ectopically produced LEP might bind to the same target genes as the AP2/EREBP family members, which normally are active in these processes. Consequently, LEP would block these target sites for other transcription factors. It is also possible that LEP activates these target genes and thus disturbs their normal transcriptional regulation.

\section{Activation tagging}

An altered development as observed for the let mutant has not previously been described for dicotyledonous plants and this clearly shows that certain (dominant) phenotypes can only be provoked by an increased and/or ectopic expression of tagged genes. Therefore, the use of activator elements in gene tagging studies is complementary to loss-of-function studies, because it provides the opportunity to generate new types of gain-offunction mutants. The let mutant could only be isolated because the activation tagging of $L E P$ caused a slight alteration in $L E P$ expression pattern. Constitutive overexpression by placing the $L E P$ coding region under the transcriptional control of the CaMV $35 \mathrm{~S}$ promoter, a procedure that is normally used to study the effect of overexpression of a gene of interest, resulted in severely deformed plants which were not viable. This indicates that the use of activator tags containing only enhancer elements has the advantage over constitutive promoters that such elements can provoke tissue-specific upregulation of activation-tagged genes, which potentially reveals more about the function of the tagged gene than constitutive overexpression.

Further studies on the role of LEP in leaf blade formation, the regulation of LEP expression, the possible interaction of LEP with other transcription factors and the identification of genes that are regulated by LEP is necessary to determine the exact role of LEP during plant development. In addition, the $L E P$ gene and its homologs from other species may be engineered to increase the yield of crop plants with petiolated leaves.

We would like to thank Gerda Lamers and Tonny RegensburgTuink for assistance with the anatomical analysis; Nicolas Baumberger and members from the Schneitz lab for assistance with the in situ hybridisation procedure and RT-PCR; Jean-Jacques Pittet for assistance with the digital processing of the figures; and Drs Remko Offringa, Bert van der Zaal and Christoph Ringli for critical reading of the manuscript. E. vdG. was supported by a grant from the Swiss National Science Foundation (31-51055.97).

\section{REFERENCES}

Bowman, J. L. (2000). The $Y A B B Y$ gene family and abaxial cell fate. Curr. Opin. Plant Biol. 3, 17-22.

Donnelly, P. M., Bonetta, D., Tsukaya, H., Dengler, R. E. and Dengler, N. G. (1999). Cell cycling and cell enlargement in developing leaves of Arabidopsis. Dev. Biol. 215, 407-419.

Gleave, A. P. (1992). A versatile binary vector system with a T-DNA organisational structure conducive to efficient integration of cloned DNA into the plant genome. Plant Mol. Biol. 20, 1203-1207.

McConnell, J. R. and Barton, M. K. (1998). Leaf polarity and meristem formation in Arabidopsis. Development 125, 2935-2942.

McHale, N. A. (1992). A nuclear mutation blocking initiation of the lamina in leaves of Nicotiana sylvestris. Planta 186, 355-360.

McHale, N. A. (1993). LAM-1 and FAT genes control development of the leaf blade in Nicotiana sylvestris. Plant Cell 5, 1029-1038.

McHale, N. A. and Marcotrigiano, M. (1998). LAM1 is required for dorsoventrality and lateral growth of the leaf blade in Nicotiana. Development 125, 4235-4243. 
Memelink, J., Swords, K. M. M., Staehelin, L. A. and Hoge, J. H. C. (1994). Southern, northern and western blot analysis. In Plant Molecular Biology Manual (ed. S. B. Gelvin and R. A. Schilperoort), pp.1-23. Dordrecht: Kluwer.

Moose, S. P. and Sisco, P. H. (1996). GLOSSY15, an APETALA2-like gene from maize that regulates leaf epidermal cell identity. Genes Dev. 10, 3018-3027.

Ohme-Takagi, M. and Shinshi, H. (1995). Ethylene-inducible DNA binding proteins that interact with an ethylene-responsive element. Plant Cell 7, 173-182.

Okamuro, J. K., Caster, B., Villarroel, R., Van Montagu, M. and Jokufu, K. D. (1997). The AP2 domain of APETALA2 defines a large new family of DNA binding proteins in Arabidopsis. Proc. Natl. Acad. Sci. USA 94, 7076-7081.

Pickett, F. B., Champagne, M. M. and Meeks-Wagner, D. R. (1996). Temperature-sensitive mutations that arrest Arabidopsis shoot development. Development 122, 3799-3807.

Poethig, R. S. (1997). Leaf morphogenesis in flowering plants. Plant Cell $\mathbf{9}$, 1077-1087.

Rechsteiner, M. and Rogers, S. W. (1996). PEST sequences and regulation by proteolysis. Trends Biochem. Sci. 21, 267-271.

Riechmann, J. L. and Meyerowitz, E. M. (1998). The AP2/EREBP family of plant transcription factors. Biol. Chem. 379, 633-646.

Rogers, S., Wells, R. and Rechsteiner, M. (1986). Amino acid sequences common to rapidly degraded proteins: the PEST hypothesis. Science 234, 364-368.

Sato, S., Kotani, H., Nakamura, Y., Kaneko, T., Asamizu, E., Fukami, M., Miyajima, N. and Tabata, S. (1997). Structural analysis of Arabidopsis thaliana chromosome 5. I. Sequence features of the $1.6 \mathrm{Mb}$ regions covered by twenty physically assigned P1 clones. DNA Res. 4, 215-230.

Schneitz, K., Hulskamp, M., Kopczak, S. D. and Pruitt, R. E. (1997). Dissection of sexual organ ontogenesis: a genetic analysis of ovule development in Arabidopsis thaliana. Development 124, 1367-1376.

Torii, K. U., Mitsukawa, N., Oosumi, T., Matsuura, Y., Yokoyama, R., Whittier, R. F. and Komeda, Y. (1996). The Arabidopsis ERECTA gene encodes a putative receptor kinase with extracellular leucine-rich repeats. Plant Cell 8, 735-746.

Tsukaya, H., Tsuge, T. and Uchimiya, H. (1994). The cotyledon: a superior model for studies of leaf development. Planta 195, 309-312.
Tsukaya, H. (1995). Developmental genetics of leaf morphogenesis in dicotyledonous plants. J. Plant Res. 108, 407-416.

van der Graaff, E., den Dulk-Ras, A. and Hooykaas, P. J. J. (1996) Deviating T-DNA transfer from Agrobacterium tumefaciens to plants. Plant Mol. Biol. 31, 677-681.

van der Graaff, E. and Hooykaas, P. J. J. (1996). Improvements in the transformation of Arabidopsis thaliana C24 leaf-discs by Agrobacterium tumefaciens. Plant Cell Rep. 15, 572-577.

van der Graaff, E. and Hooykaas, P. J. J. (1998). Transformation of Arabidopsis thaliana $\mathrm{C} 24$ leaf discs by Agrobacterium tumefaciens. In Arabidopsis Protocols (ed. J. Martinez-Zapater and J. Salinas), pp.245-258. Totowa, NJ: Humana Press.

Van Lijsebettens, M. and Clarke, J. (1998). Leaf development in Arabidopsis. Plant Physiol. Biochem. 36, 47-60.

van Slogteren, G. M. S., Hoge, J. H. C., Hooykaas, P. J. J. and Schilperoort, R.A. (1983). Clonal analysis of heterogenous crown gall tumor tissues induced by wildtype and shooter mutant strains of Agrobacterium tumefaciens - expression of T-DNA genes. Plant Mol. Biol. 2, 321-333.

Vergunst, A. C., de Waal, E. C. and Hooykaas, P. J. J. (1998). Root transformation by Agrobacterium tumefaciens. In Arabidopsis Protocols. (ed. J. Martinez-Zapater and J. Salinas), pp. 227-244. Totowa, NJ: Humana Press.

Vielle-Calzada, J.-P., Thomas, J., Spillane, C., Coluccio, A., Hoeppner, M. A. and Grossniklaus, U. (1999). Maintenance of genomic imprinting at the Arabidopsis MEDEA locus requires zygotic DDM1 activity. Genes Dev. 13, 2971-2982.

Waites, R. and Hudson, A. (1995). PHANTASTICA: a gene required for dorsoventrality of leaves in Antirrhinum majus. Development 121, 2143-2154.

Waites, R., Selvadurai, H. R. N., Oliver, I. R. and Hudson, A. (1998). The PHANTASTICA gene encodes a MYB transcription factor involved in growth and dorsoventrality of lateral organs in Antirrhinum. Cell $\mathbf{9 3}$ 779-789.

Weigel, D. (1995). The APETALA2 domain is related to a novel type of DNA binding domain. Plant Cell 7, 388-389.

Wilson, K., Long, D., Swinburne, J. and Coupland, G. (1996). A Dissociation insertion causes a semidominant mutation that increases expression of TINY, an Arabidopsis gene related to APETALA2. Plant Cell 8, 659-671. 